\title{
Cosmological analogies in the search for new physics in high-energy collisions
}

\author{
Miguel-Angel Sanchis-Lozano, ${ }^{1, *}$ Edward K. Sarkisyan-Grinbaum, ${ }^{2,3, \dagger}$ \\ Juan-Luis Domenech-Garret $\oplus^{4, \$}$ and Nicolas Sanchis-Gual $\oplus^{5,8}$ \\ ${ }^{1}$ Instituto de Física Corpuscular (IFIC) and Departamento de Física Teórica Centro Mixto \\ Universitat de València-CSIC, Dr. Moliner 50, E-46100 Burjassot, Spain \\ ${ }^{2}$ Experimental Physics Department, CERN, 1211 Geneva 23, Switzerland \\ ${ }^{3}$ Department of Physics, University of Texas at Arlington, Texas 76019, USA \\ ${ }^{4}$ Departamento de Física A.I.A.N., Universidad Politécnica de Madrid, E-28040 Madrid, Spain \\ ${ }^{5}$ Centro de Astrofísica e Gravitação-CENTRA, Departamento de Física, Instituto Superior Técnico-IST, \\ Universidade de Lisboa-UL, Avenida Rovisco Pais 1, 1049-001 Lisboa, Portugal
}

(Received 17 June 2020; accepted 31 July 2020; published 11 August 2020)

\begin{abstract}
In this paper, analogies between multiparticle production in high-energy collisions and the time evolution of the early Universe are discussed. A common explanation is put forward under the assumption of an unconventional early state: a rapidly expanding universe before recombination (last scattering surface), followed by the cosmic microwave background, later evolving up to present days, versus the formation of hidden/dark states in hadronic collisions followed by a conventional QCD parton shower yielding final-state particles. In particular, long-range angular correlations are considered pointing out deep connections between the two physical cases potentially useful for the discovery of new physics.
\end{abstract}

DOI: 10.1103/PhysRevD.102.035013

\section{INTRODUCTION}

The study of correlations has decisively contributed to the advancement of scientific knowledge in all branches of physics, from condensed matter and quantum information to particle physics and cosmology. In the latter case, the systematic study of correlations was considered in the context of the large structure of the Universe [1]. In this sense, the homogeneity, thus long-range angular correlations of the cosmic microwave background (CMB) across the sky seen by the WMAP and Planck missions [2,3], strongly supports an inflationary era of the early Universe [4,5]. This proposal gave birth to a new paradigm in astrophysics and cosmology, ultimately leading to the standard cosmological model $(\Lambda \mathrm{CDM})$.

Similarly, the study of angular correlations in highenergy collisions has traditionally been a common tool to understand multiparticle production in particle collisions beginning with early studies of cosmic rays through to

\footnotetext{
${ }^{*}$ Miguel.Angel.Sanchis@ific.uv.es

†dward.Sarkisyan-Grinbaum@cern.ch

*domenech.garret@upm.es

§nicolas.sanchis@tecnico.ulisboa.pt
}

Published by the American Physical Society under the terms of the Creative Commons Attribution 4.0 International license. Further distribution of this work must maintain attribution to the author(s) and the published article's title, journal citation, and DOI. Funded by SCOAP . current investigations at the LHC. In earlier papers, we show that one consequence of the production of a new still unknown stage of matter in high-energy hadronic collisions is to enhance long-range angular correlations among finalstate particles [6-8]. This conclusion bears a certain resemblance with the observed small temperature fluctuations of the $\mathrm{CMB}$ requiring an inflationary period right after the big bang.

As is well known, analogies between different fields of knowledge have traditionally played an important role in the advance of science. A paradigmatic example in physics is provided by the analogy between superconductivity in condensed matter physics, and the vacuum screening currents leading to the Higgs mechanism in elementary particle physics [9]. Although the physical origin may be totally distinct (Cooper electron pairs versus a Higgs quantum field current), a mapping can be established between the equations governing both processes, as well as some specific relations between the theory parameters. Actually, such an analogy proved to be a useful guide for getting a deeper understanding of the origin of mass and further developments of the electroweak theory.

The main goal of this paper is showing an analogy between the cosmic evolution of the Universe and multiparticle production in high-energy collisions, as well as its consequences as a new way of hunting hidden/dark matter at the LHC and other future facilities. A caveat is in order however: in the former case, there is only one universe 
(ours) to be observed, while in the latter a large number of independent collisions are statistically considered altogether. This difference should not alter the main consequences of our analogy.

\section{ANGULAR CORRELATIONS IN MODERN COSMOLOGY}

According to the firstly postulated big bang theory, the angular scale of the horizon on the last scattering surface (when the $\mathrm{CMB}$ was emitted) should be $\theta \simeq 1^{\circ}$ [10]. This implies that strong temperature inhomogeneities should show up above this scale in contrast to real measurements which reveal an extremely isotropic and homogeneous microwave background. To solve this problem, an inflationary era in the very early Universe was proposed, flattening all fluctuations up to very large opening angles covering the entire celestial sphere.

On the other hand, the emergence of large-scale features in the $\mathrm{CMB}$ are attributed to density fluctuations in the early Universe evolving into the large-scale structure as we see today. In fact, it is common wisdom that such small temperature fluctuations (of the order of $10^{-5} \mathrm{~K}$ ) are the seeds of the current observed galaxy distributions, galaxy clusters and higher macrostructures of our Universe.

Two categories of temperature fluctuations observed in the $\mathrm{CMB}$ can be distinguished according to the universal time evolution: (a) primary anisotropies, prior to decoupling, and (b) secondary anisotropies developing as the CMB propagates from the surface of the last scattering to the observer. The former include temperature inhomogeneities due to photon propagation under metric fluctuations, the so-called Sachs-Wolfe (SW) effect. This effect shows up at rather large angles, i.e., for $\theta \gg 1^{\circ}$, where $\theta$ stands for the angular separation of different directions in the present sky. Moreover, the primitive plasma also underwent acoustic oscillations prior to decoupling associated to a typical angular scale $\theta \lesssim 1^{\circ}$.

On the other hand, once photons decoupled from baryons after recombination, the CMB propagated through a large structure where the gravitational and intercluster gas which are not be necessarily isotropic nor homogeneous on small spatial scales. Examples of such secondary anisotropies of the CMB include the Sunyaev-Zeldovich effect due to thermal electrons and the integrated SW effect, induced by the time variation of gravitational potentials. These effects are mainly expected to produce temperature fluctuations on arc-minute scales. In this work, we shall consider them altogether under a common parametrization of very-short-range correlations.

Let us emphasize that what matters in our analogy on angular correlations is the existence of two well differentiated steps in the evolution of the Universe, before and after recombination. Therefore, rather than modeling an inflationary epoch in the primitive Universe, we sill assume a linearly expanding universe whose scale factor reads: $a(t)=t / t_{f}$, where $t$ stands for the universal time and $t_{f}$ for the time elapsed since the big bang to present. In Refs. [11,12], a model of this kind was proposed and developed to explain the observed correlations of the $\mathrm{CMB}$. Then, the maximum fluctuation size at any given time $t$ can be estimated as $\lambda_{\max }=2 \pi R(t)$ with $R$ as the cosmic horizon radius.

Following the reasoning of [11], the comoving distance to the last scattering surface (at recombination time $t_{\text {rec }}$ ) reads

$$
r_{\mathrm{rec}}=c t_{f} \int_{t_{\mathrm{rec}}}^{t_{f}} \frac{d t}{t}=c t_{f} \ln \left[\frac{t_{f}}{t_{\mathrm{rec}}}\right] .
$$

Thus, the maximum angular size $\theta_{0}$ of fluctuations associated to the CMB emitted at $t_{\text {rec }}$ is given by

$$
\theta_{0}=\frac{\lambda_{\max }}{R\left(t_{\mathrm{rec}}\right)}
$$

where

$$
R\left(t_{\text {rec }}\right)=a\left(t_{\text {rec }}\right) r_{\text {rec }}=c t_{\text {rec }} \ln \left[\frac{t_{f}}{t_{\text {rec }}}\right] .
$$

Finally, one gets

$$
\theta_{0} \sim \frac{2 \pi}{\ln \left[t_{f} / t_{\mathrm{rec}}\right]} \simeq \frac{\pi}{5}
$$

where the numerical estimate corresponds to $t_{f}=13.8 \mathrm{Gyr}$ and $t_{\text {rec }}=3.8 \times 10^{5} \mathrm{yr}$. This value roughly agrees with the curve determined from Planck data $(\simeq \pi / 3)$ as shown in [11]. Let us remark that Eq. (4) is considered here as a simple indicator of long-range angular correlations in the $\mathrm{CMB}$, to be later "translated" to high-energy hadronic collisions.

\section{NEW PHYSICS FROM AZIMUTHAL CORRELATIONS IN HIGH-ENERGY COLLISIONS}

Long-range angular correlations (both in pseudorapidity and azimuth) also show up in multiparticle production in both $p p$ and heavy-ion collisions [13]. From general arguments based on causality, such long-range correlations can be traced back to the very early times after the primary parton-parton hadronic interactions. As stressed in Ref. [6], if the parton shower were to be altered by the presence of a nonconventional state of matter, final-state particle correlations should be sensitive to it.

We focus on strongly interacting dark sectors arising in a wide variety of new physics scenarios like e.g., the hidden valley [14-16]. Hidden valley models predict the existence of a hidden/dark sector connected to the Standard Model (SM) of particle physics through heavy mediators via different mechanisms (tree-level, higher loop 


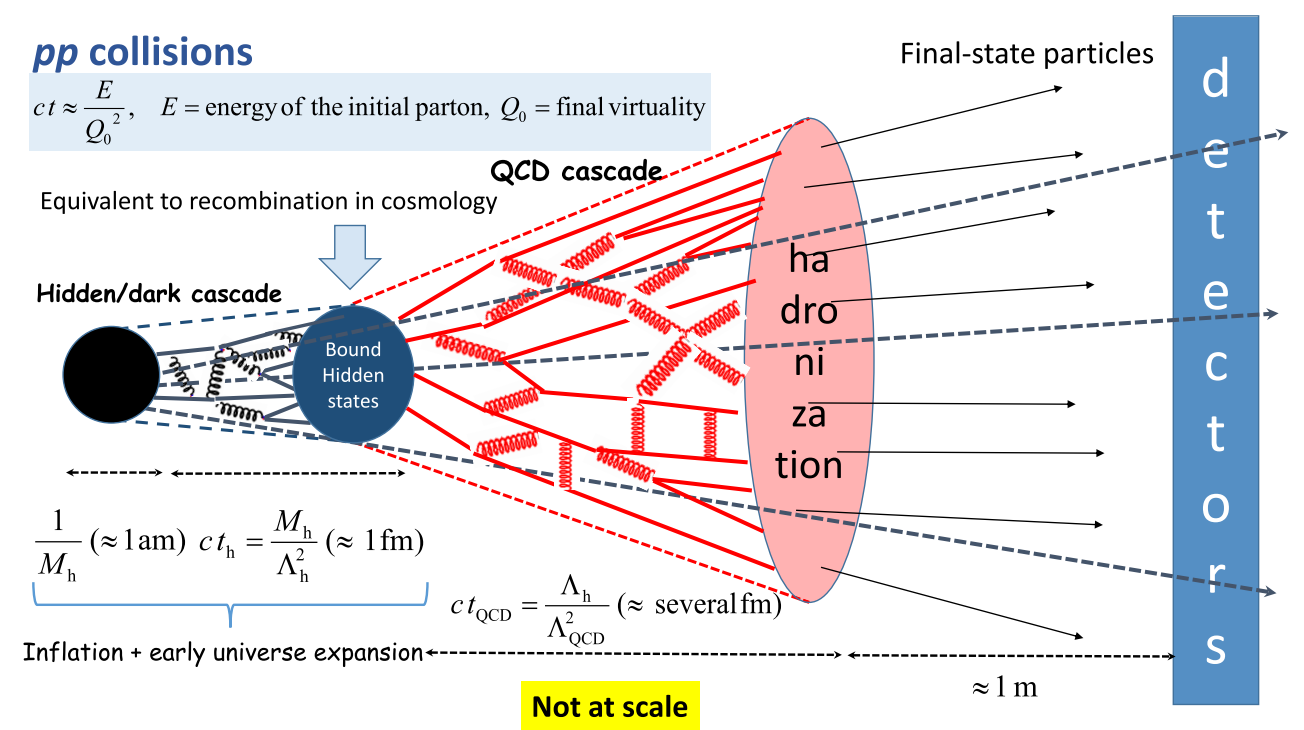

FIG. 1. Time evolution of the parton shower in high-energy collisions with formation of an initial hidden stage of matter evolving into bound states (equivalent to recombination in cosmology) after a time $t$, followed by a decay back to SM quarks and gluon partons lasting about $t_{\mathrm{QCD}}$. There could also be hidden particles not decaying back to SM and therefore, not detected, represented as dashed arrows.

diagrams). One of the most interesting situations from a phenomenological viewpoint corresponds to a QCDinspired scenario with a hidden (running) coupling constant and a confinement scale $\Lambda_{\mathrm{h}}$. Then the hidden/dark quarks, which could be much lighter than the energy scale set by the heavy mediator $M_{\mathrm{h}}$, can form bound states at $\Lambda_{\mathrm{h}}$ as hadrons in QCD. Usually the masses of the hidden sector particles are assumed to lie below the electroweak scale, while the mediators may have $\mathrm{TeV}$-scale masses. Therefore, it seems quite natural to expect large hierarchies between $\Lambda_{\mathrm{h}}$ and the hidden quark masses $m_{\mathrm{h}}$ [17],

$$
m_{\mathrm{h}} \sim \Lambda_{\mathrm{h}} \ll M_{\mathrm{h}},
$$

where the condition on the relative value of $\Lambda_{\mathrm{h}} \sim m_{\mathrm{h}}$ can be taken quite loosely without changing the main conclusions of this paper.

In our numerical estimates, we assume that the strongly coupled hidden sector includes some families of hidden quarks that bind into hidden hadrons at energies below $\Lambda_{\mathrm{h}} \sim O(10) \mathrm{GeV}$, playing a similar role as $\Lambda_{\mathrm{QCD}}$ in the conventional strong interaction. Such a simplified picture is compatible with the expected walking behavior requiring a strong coupling over a large energy window before reaching $\Lambda_{\mathrm{h}}$, thereby yielding a large number of hidden partons and ultimately, high multiplicity events where the primary energy is democratically shared by final-state particles.

On the other hand, the SM sector could feebly couple to the hidden sector (and the equivalent hadronic hidden particles and states) with a substantial freedom in the form of the portal interaction: via a tree-level neutral $Z^{\prime}$ or higher-order loops involving particles with charges of both
SM and hidden sectors. In fact, for some (reasonable) values of the parameter space in hidden valley models, hidden particles can promptly decay back into SM particles, altering the subsequent conventional parton shower [16] and yielding (among others [17]) observable consequences, e.g., extremely long-range correlations especially in azimuthal space [7].

The maximum length, i.e., time, ${ }^{1}$ of a parton shower initiated by a parton, down to a low virtuality scale $Q_{0}$, can be estimated as [18]

$$
L_{\max } \simeq \frac{E}{Q_{0}^{2}},
$$

where $E$ stands for the typical energy of the parton cascade; $Q_{0}$ is expected to be of the order of $\Lambda_{\mathrm{QCD}}$ for a conventional QCD cascade and of the order of $\Lambda_{\mathrm{h}}$ for a dark cascade.

In Fig. 1, we show pictorially the foreseen evolution after a primary hard parton-parton interaction producing a hidden shower as a first stage of the cascade ultimately yielding final-state particles via a QCD parton cascade. Three steps can be distinguished:

(i) Production of heavy mediators of mass $\mathcal{O}\left(10^{3}\right) \mathrm{GeV}$ in the primary partonic collision. This is assumed to occur at a tiny fraction of a second $(\simeq 1 \mathrm{am} / c)$, fixed by the energy scale $M_{\mathrm{h}}$.

(ii) Hidden shower and formation of hidden bound states (equivalent to recombination in cosmology),

\footnotetext{
${ }^{1}$ Hereafter, natural units, $c=\hbar=1$, will be used unless otherwise stated.
} 
at typical time $t_{\mathrm{h}}=M_{\mathrm{h}} / Q^{2}$, where $Q^{2}$ stands for the virtuality of the hidden shower. Assuming that $Q^{2}$ is of the order of $\Lambda_{\mathrm{h}}^{2} \simeq 100 \mathrm{GeV}^{2}$, one gets a time scale of the order of $1 \mathrm{fm} / c$.

(iii) Once the hidden bound states (or a part of them) decay back to QCD partons (quarks and gluons), a "conventional" cascade takes place with typical time $t_{\mathrm{QCD}}=\Lambda_{\mathrm{h}} / \Lambda_{\mathrm{QCD}}^{2} \sim$ several fm $/ c$, where we have assumed that the typical energy of the now conventional parton shower is provided by $\Lambda_{\mathrm{h}} \sim m_{\mathrm{h}}$.

\section{COSMOLOGICAL ANALOGIES}

Since the successful running of the heavy-ion program at the LHC, it has become popular to compare the evolution of the Universe, some seconds after the big bang, with the formation of very dense matter at high temperature (presumably forming a soup of quarks and gluons) in hadronic collisions. It has even become customary talking somewhat loosely about a "little big bang" at the LHC. Moreover, such a parallelism between the space-time developments of heavy-ion collisions and the early Universe has been considered beyond purely outreach purposes as a source of physical inspiration; see, e.g., Ref. [19]. In a recent paper [20], the authors established a correspondence between high-energy collions at future $e^{+} e^{-}$colliders (ILC and CLIC) and the CMB map.

It should be mentioned that the physics underlying angular correlations is completely different in the two cases: the cosmological evolution is fundamentally described by general relativity, whereas the parton cascade evolution in high-energy collisions is essentially governed by conventional or hidden strong interaction dynamics. However, on the one hand, they share a common fact put forward to explain long-range correlations: a rapidly growing initial state. On the other hand, the typical values of the angular scales are (by coincidence) quite similar as we shall see. Indeed, primary long-range angular correlations are of the order of 1 radian, while secondary scales lie 1 order of magnitude or more below. Such a numerical concordance of scales, together with the fact that the time evolution in both cases is not continuous but rather involves different well-defined steps, makes a connection between the two cases.

Now, turning to high-energy collisions and naively applying the same expression (3) used for a particular cosmological model, setting $t_{\mathrm{h}}=M_{\mathrm{h}} / \Lambda_{\mathrm{h}}^{2}, t_{\mathrm{QCD}}=\Lambda_{\mathrm{h}} / \Lambda_{\mathrm{QCD}}^{2}$ and $t_{f} \simeq t_{\mathrm{h}}+t_{\mathrm{QCD}}$ (see Fig. 1), we get for the maximal azimuthal correlation angle,

$$
\phi_{0} \simeq \frac{2 \pi}{\ln \left[t_{f} / t_{\mathrm{h}}\right]} \simeq \pi
$$

for $\Lambda_{\mathrm{h}} \simeq 10 \mathrm{GeV}$ and $M_{\mathrm{h}} \simeq 1 \mathrm{TeV}$ as reference values. As already commented, this result points at very longrange correlations emerging in a "universe" under the above-mentioned evolution conditions. ${ }^{2}$ Such an orderof-magnitude estimate is in agreement with our earlier estimates [8] about the expected long-range correlation length in azimuthal space arising from new physics. Here, we explore further the analogies between cosmic evolution and hadronic evolution under the presence of a hidden sector on top of the QCD shower. Note that temperature fluctuations are supposed to be the seeds of the current observed galaxy distributions, galaxy clusters, and higher macrostructures of our Universe. Similarly, hidden (bound) states would act as the seeds of clusters (jets in a broad sense) of final-state particles.

The two-point correlation function is defined in many different (though essentially related) ways in the literature. For example, in the case of the temperature correlations of the CMB seen in directions $\vec{n}_{1}$ and $\vec{n}_{2}$ of the sky, the twopoint correlation function can be written as the ensembleaverage product,

$$
C_{2}(\cos \Delta \theta)=\left\langle T\left(\vec{n}_{1}\right) T\left(\vec{n}_{2}\right)\right\rangle,
$$

where isotropy and homogeneity of space have been assumed, and $\cos \Delta \theta=\vec{n}_{1} \cdot \vec{n}_{2}$. It measures the conditional probability of having two $\mathrm{CMB}$ temperatures in the sky plane differing by $\Delta \theta=\theta_{1}-\theta_{2}$. Furthermore, the threepoint angular correlation function is defined as [21]

$$
C_{3}\left(\cos \Delta \theta_{12}, \cos \Delta \theta_{13}\right)=\left\langle T\left(\vec{n}_{1}\right) T\left(\vec{n}_{2}\right) T\left(\vec{n}_{3}\right)\right\rangle,
$$

where now three different directions in the sky are labeled by three vectors $\vec{n}_{i}, i=1,2,3$, and $\Delta \theta_{12}=\theta_{1}-\theta_{2}$, $\Delta \theta_{13}=\theta_{1}-\theta_{3}$. Note that actually only two angular differences are independent, here chosen $\Delta \theta_{12}$ and $\Delta \theta_{13}$, so that $\Delta \theta_{23}=\theta_{2}-\theta_{3}=\Delta \theta_{13}-\Delta \theta_{12}$.

Notice that already since some time ago, the study of three-point correlations has been recognized as a powerful probe of the origin and evolution of structures of the Universe; see, e.g., [22-24]. Specifically, non-Gaussian contributions to cosmological correlations should play a leading role in understanding the physics of the early Universe, when primordial seeds for large-scale structures were created, and their subsequent growth at later times. Interestingly, the correlation function method was recently proposed in Ref. [25] to distinguish between quantum and classical primordial fluctuations in a sense close to our consideration.

In high-energy collisions, the two-particle correlation function $C_{2}\left(\phi_{1}, \phi_{2}\right)$ is similarly defined, where $\phi_{i}$ stands for the azimuthal emission angle of particle $i$ measured on the transverse plane of a reference frame whose $z$ axis corresponds to the beams direction. Under rotation symmetry, the two-point correlation function actually

\footnotetext{
${ }^{2}$ Of course, the actual situation in high-energy particle collisions at colliders is not the same as in an expanding universe, where space itself is being created as the expansion goes on. Nevertheless, one can still keep in mind the picture of a growing particle horizon to be identified somehow with the radius of a growing sphere containing the developing parton cascade inside.
} 

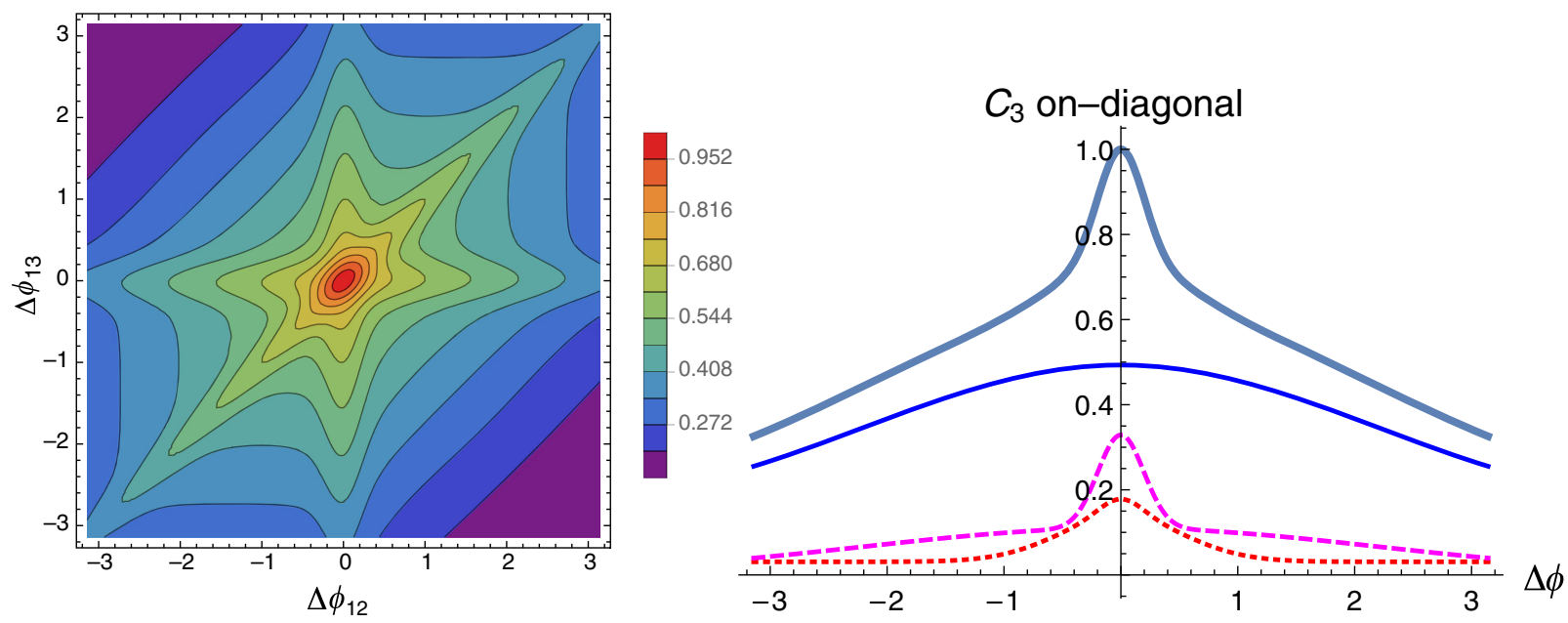

FIG. 2. Left: Contour plot of the three-particle correlation function $C_{3}\left(\Delta \phi_{12}, \Delta \phi_{13}\right)$ of a three-step cascade with an initial long-range contribution from a hidden sector. Right: Diagonal projection of $C_{3}\left(\Delta \phi_{12}, \Delta \phi_{13}\right)$, where the peak at $\Delta \phi_{1}=\Delta \phi_{2}=0$ is normalized to unity. The dotted (red), dashed (magenta) and thin solid (blue) curves show the contributions from one, two, and three hidden particles, respectively. The weighted sum is shown by the thick (turquoise) curve. Plots are taken from our work [7].

depends only on the azimuthal difference $\Delta \phi=\phi_{1}-\phi_{2}$, i.e., $C_{2}\left(\phi_{1}, \phi_{2}\right)=C_{2}(\Delta \phi)$.

Again, higher-order correlations are useful as well to get a deeper insight into multiparticle dynamics in hadronic collisions [26]. A dependence of the correlations on angular differences is expected too, e.g., the threeparticle azimuthal correlation function $C_{3}\left(\Delta \phi_{12}, \phi_{13}\right)$, where $\Delta \phi_{12}=\phi_{1}-\phi_{2}, \Delta \phi_{13}=\phi_{1}-\phi_{3}$. Let us point out that three-point/particle correlations constitute the lowestorder statistical tool to check the non-Gaussianity of distributions. Furthermore, they can place strong constraints on underlying clustering structures, thereby becoming specially suited to uncover new physics in multiparticle production in particle collisions as stressed in [27]. Generally speaking, three-particle angular correlations may suggest the formation of primary clusters [28]; larger cluster sizes imply stronger three-particle correlations.

In our approach to multiparticle production, correlations are modeled by using Gaussian distributions for either cluster and final state particle production in high-energy collisions [29]. Thereby, we make use of Gaussian widths to parametrize the typical correlation lengths in the different steps of hadron production.
As shown in [7], the three-particle correlation function can be written as

$$
\begin{aligned}
C_{3}\left(\Delta \phi_{12}, \Delta \phi_{13}\right)= & \frac{1}{\left\langle N_{\mathrm{h}}\right\rangle^{2}} h^{(1)}\left(\Delta \phi_{12}, \Delta \phi_{13}\right) \\
& +\frac{1}{\left\langle N_{\mathrm{h}}\right\rangle} h^{(2)}\left(\Delta \phi_{12}, \Delta \phi_{13}\right) \\
& +h^{(3)}\left(\Delta \phi_{12}, \Delta \phi_{13}\right)
\end{aligned}
$$

where each term on the rhs represents the correlations due to one, two, and three initial sources of hidden particles, indicated by the upper index of the $h$ functions, produced in the same initial partonic interaction; $\left\langle N_{\mathrm{h}}\right\rangle$ denotes the mean number of hidden sources per collision. Note that the $h$ functions include the angular dependence due to all possible correlations, namely, particle correlations in clusters, cluster correlations, and hidden source correlations.

The bigger long-range correlations for the three-particle correlation function $C_{3}\left(\Delta \phi_{12}, \Delta \phi_{13}\right)$ in a three-step cascade are given by the $h^{(3)}\left(\Delta \phi_{12}, \Delta \phi_{13}\right)$ term, associated to three initial hidden/dark particles,

$$
\begin{aligned}
h^{(3)}\left(\Delta \phi_{12}, \Delta \phi_{13}\right) \sim & \exp \left[-\frac{\left(\Delta \phi_{12}\right)^{2}+\left(\Delta \phi_{13}\right)^{2}-\Delta \phi_{12} \Delta \phi_{13}}{3 \delta_{\mathrm{h} \phi}^{2}+\delta_{\mathrm{h} \phi}^{2}}\right]+\exp \left[-\frac{\left(\Delta \phi_{12}\right)^{2}}{2\left(2 \delta_{\mathrm{c} \phi}^{2}+\delta_{\mathrm{h} \phi}^{2}\right)}\right] \\
& +\exp \left[-\frac{\left(\Delta \phi_{13}\right)^{2}}{2\left(2 \delta_{\mathrm{c} \phi}^{2}+\delta_{\mathrm{h} \phi}^{2}\right)}\right]+\exp \left[-\frac{\left(\Delta \phi_{12}\right)^{2}+\left(\Delta \phi_{13}\right)^{2}-2 \Delta \phi_{12} \Delta \phi_{13}}{2\left(2 \delta_{\mathrm{c} \phi}^{2}+\delta_{\mathrm{h} \phi}^{2}\right)}\right] .
\end{aligned}
$$

Here, $\delta_{\mathrm{h} \phi}$ and $\delta_{\mathrm{c} \phi}$ represent the expected correlation length due to the first and second steps in the evolution of the parton cascade using a simplified model. In turn, correlations of particles from clusters are parametrized by $\delta_{\phi}$, which can be referred to as the cluster decay width in the transverse plane (see [7,29]). The full set of expressions for the three-particle correlation function $C_{3}\left(\Delta \phi_{12}, \Delta \phi_{13}\right)$ in a three-step cascade process can be found in [7]. 

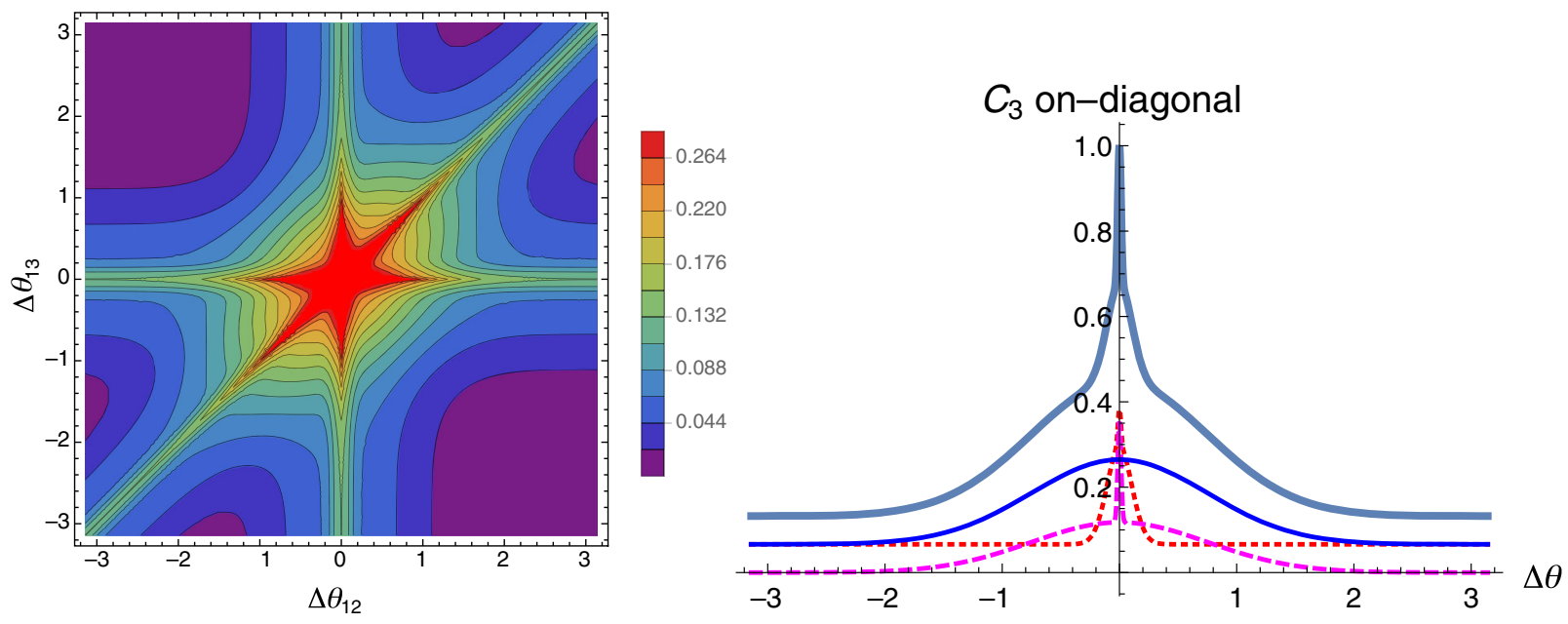

FIG. 3. Left: Contour plot of the three-point correlation function $C_{3}\left(\Delta \theta_{12}, \Delta \theta_{13}\right)$ in the cosmological case using a toy model to take into account different sources of short-range and long-range correlations. Right: Diagonal projection of $C_{3}\left(\Delta \theta_{12}, \Delta \theta_{13}\right)$ showing the different contributions in analogy to Fig. 2.

The plots of the three-particle correlation function obtained from numerical estimates of a three-step cascade with an initial long-range contribution from a hidden sector are shown in Fig. 2. The left panel shows a typical (spiderweb) structure of the three-particle correlations in a two-dimensional $\left(\Delta \phi_{12}, \Delta \phi_{13}\right)$ plot. The right panel shows its projection along the diagonal $\Delta \phi_{12}=\Delta \phi_{13}$. The latter plot stands for three-particle correlations disentangling the diverse sources of short-range and long-range angular correlations.

Turning to the cosmological analogy, we apply the same analysis of angular correlations in the CMB as employed in high-energy collisions with the following correspondence in our Gaussian parametrization: $\delta_{\mathrm{h} \phi} \rightarrow \delta_{\mathrm{h} \theta}$ for long-range correlations, $\delta_{\mathrm{c} \phi} \rightarrow \delta_{\mathrm{c} \theta}$ for short-range correlations, and $\delta_{\phi} \rightarrow \delta_{\theta}$ for very-short-range (within-cluster) correlations, respectively.

As commented above, two kinds of anisotropies can be distinguished in the CMB:

(1) Primary anisotropies produced prior to recombination/decoupling, yielding rather long-range correlations, mainly due to the SW effect. In our parametrization, it corresponds to $\delta_{\mathrm{h} \theta} \simeq 1 \mathrm{rad}$ [see Eq. (4)]. Also, baryon acoustic oscillations of the plasma contribute to anisotropies but at a smaller scale, implying $\delta_{\mathrm{c} \theta} \lesssim 1^{\circ}$.

(2) Secondary anisotropies developing as the CMB propagates from the last scattering surface to the present observer leading to very-short-range angular correlations. The set of such effects yields in our parametrization to $\delta_{\theta} \ll 1^{\circ}$.

Notice that the angular scales showing up as anisotropies in the CBM are not too much different from the expected azimuthal scales stemming from multiparticle production via a hidden sector on top of the partonic shower in highenergy collisions [7].

Figure 3 shows the three-point correlation function $C_{3}\left(\Delta \theta_{12}, \Delta \theta_{13}\right)$ plots for cosmological estimates using a simple model which takes into account the overall sources of short-range and long-range correlations. The left panel shows the two-dimensional plot of $C_{3}\left(\Delta \theta_{12}, \Delta \theta_{13}\right)$ as a function of $\Delta \theta_{12}$ and $\Delta \theta_{13}$, while the right panel shows the on diagonal projection of the function. This figure is analogous to Fig. 2 for high-energy collisions. Again, the three-point correlation function is arbitrarily normalized to unity at $\Delta \theta_{12}=\Delta \theta_{13}=0$, since we are here interested rather in disentangling the different sources to angular correlations. Of course, a more realistic study should incorporate the absolute normalization and relative weights using a more detailed model. Note that the $h\left(\Delta \theta_{12}, \Delta \theta_{13}\right)$ functions, i.e., the equivalent cosmological terms in Eq. (10) for high-energy collisions, are similarly sensitive to very-short-range, short-range and long-range correlations, respectively. This is an important result of our work.

By comparing Figs. 2 and 3, an equivalent structure can be appreciated in both panels as expected from the common existence of angular short-range and long-range correlations, no matter their physical origin. Again, as in the case of high-energy hadronic collisions, several distinct correlation scales clearly show up: short-range correlation lengths (secondary angular correlations), and a long-range correlation length (primary angular correlations) associated to the early epoch of the Universe. The diagonal projection suggests a pattern which might be useful to disentangle possible sources of angular correlations present in the CMB. Short-range and very-short-range correlations are behind the peak structure while longer correlations determine the smooth falling off. Further detailed structure can 
vary depending on the different underlying effects, but the overall behavior is expected to be quite similar.

\section{CONCLUSIONS}

In this paper, we discuss an intriguing similarity between long-range angular correlations observed in the CMB and those obtained from multiparticle production in highenergy collisions. Although the physical origin of such long-range angular correlations is completely different in the two physical situations, the analogy is supported by the following facts: the time evolution in both cases (yielding complex structures from a primitive state of matter, either galaxies or final-state particles) is not continuous but rather involves different well-defined steps, with similar angular scales. Based on this observation, a common explanation has been proposed upon the assumption of the existence of an unconventional early state: an expanding universe before recombination/decoupling (last scattering surface), where the CMB was released, evolving up to present days, versus the formation of hidden/dark states in hadronic collisions followed by a conventional QCD cascade resulting in final-state particles. Using simple modeling, we show that three-point/three-particle correlations should be a useful tool to disentangle the different contributions to short-range and long-range correlations in the Universe evolution or in multiparticle production, highlighting deep connections between both fields in the search for new physics and phenomena either at the LHC or future accelerators.

\section{ACKNOWLEDGMENTS}

This work has been partially supported by the Spanish Ministerio de Ciencia, Innovación y Universidades, under Grant No. FPA2017-84543-P and by Generalitat Valenciana under Grant No. PROMETEO/2019/113 (EXPEDITE). N. S.-G. is supported by the Fundação para a Ciência e a Tecnologia (FCT) Projects No. PTDC/FISOUT/28407/2017 and No. UID/FIS/00099/2020 (CENTRA), CERN/FIS-PAR/0027/2019, and by the European Union's Horizon 2020 research and innovation (RISE) programme H2020-MSCA-RISE-2017 Grant No. FunFiCO-777740.
[1] P. J. E. Peebles, The Large-Scale Structure of the Universe (Princeton University Press, Princeton, 1980).

[2] D. N. Spergel et al., Astrophys. J. Suppl. Ser. 148, 175 (2003).

[3] P. Ade et al. (Planck Collaboration), Astron. Astrophys. 571, A15 (2014).

[4] A. H. Guth, Phys. Rev. D 23, 347 (1981).

[5] A. D. Linde, Phys. Lett. B 108, 389 (1982).

[6] M.-A. Sanchis-Lozano, Int. J. Mod. Phys. A 24, 4529 (2009); M.-A. Sanchis-Lozano, E. K. Sarkisyan-Grinbaum, and S. Moreno-Picot, Phys. Lett. B 754, 353 (2016).

[7] M.-A. Sanchis-Lozano and E. K. Sarkisyan-Grinbaum, Phys. Lett. B 781, 505 (2018).

[8] M. A. Sanchis-Lozano and E. K. Sarkisyan-Grinbaum, Physics 1, 84 (2019).

[9] I. J. R. Aitchison and A. J. G. Hey, Gauge Theories in Particle Physics: A Practical Introduction (CRC Press, Boca Raton, FL, 2013).

[10] For a detailed discussion on cosmological effects, see: T. Padmanabhan, Theoretical Astrophysics. Vol. 3: Galaxies and Cosmology (Cambridge University Press, Cambridge, England, 2002).

[11] F. Melia, Astron. Astrophys. 561, A80 (2014).

[12] F. Melia and M. Lopez-Corredoira, Astron. Astrophys. 610, A87 (2018).

[13] For review, see e. g., K. Dusling, W. Li, and B. Schenke, Int. J. Mod. Phys. E 25, 1630002 (2016).
[14] M. J. Strassler and K. M. Zurek, Phys. Lett. B 651, 374 (2007).

[15] J. Kang and M. A. Luty, J. High Energy Phys. 11 (2009) 065.

[16] M. J. Strassler, arXiv:0806.2385.

[17] T. Cohen, M. Lisanti, H. K. Lou, and S. Mishra-Sharma, J. High Energy Phys. 11 (2017) 196.

[18] T. Renk, Phys. Rev. C 83, 024908 (2011).

[19] P. Sorensen, arXiv:0808.0503.

[20] L. Li, Y. Y. Li, T. Liu, and S. J. Xu, arXiv:2004.15013.

[21] E. Gaztañaga and J. Wagg, Phys. Rev. D 68, 021302(R) (2003).

[22] A. Gangui, F. Lucchin, S. Matarrese, and S. Mollerach, Astrophys. J. 430, 447 (1994).

[23] G. Chen and I. Szapudi, Astrophys. J. 635, 743 (2005).

[24] E. Komatsu et al., arXiv:0902.4759.

[25] D. Green and R. A. Porto, Phys. Rev. Lett. 124, 251302 (2020).

[26] W. Kittel and E. A. De Wolf, Soft Multihadron Dynamics (World Scientific, Singapore, 2005).

[27] M.-A. Sanchis-Lozano and E. Sarkisyan-Grinbaum, Phys. Rev. D 96, 074012 (2017).

[28] F. Wang, Phys. Rev. C 81, 064902 (2010).

[29] M.-A. Sanchis-Lozano and E. Sarkisyan-Grinbaum, Phys. Lett. B 766, 170 (2017). 